\title{
Workflow Management in Condor
}

\author{
Peter Couvares ${ }^{1}$, Tevfik Kosar ${ }^{2}$, Alain Roy ${ }^{1}$, Jeff Weber $^{1}$, and Kent Wenger ${ }^{1}$ \\ 1 University of Wisconsin-Madison, Computer Sciences Department \\ $\{\mathrm{pfc}$, roy, weber, wenger\}@cs.wisc.edu \\ 2 Louisiana State University, Department of Computer Science and Center for \\ Computation \& Technology \\ kosar@cct.lsu.edu
}

\subsection{Introduction}

The Condor Project began in 1988 and has evolved into a feature-rich batch system that targets high-throughput computing; that is, Condor focuses on providing reliable access to computing over long periods of time, instead of highly-tuned, high-performance computing for short periods of time or small numbers of applications.

Many Condor users have not only long-running jobs, but have complex sequences of jobs, or workflows, that they wish to run. In the late 1990s, we began development of DAGMan (or Directed Acyclic Graph Manager), which allows users to submit large workflows to Condor. As with Condor, the focus has been on reliability. DAGMan has a simple interface that allows many, but certainly not all types of workflows to be expressed. We have found, through years of experience running production workflows with our users, that solving the so-called "simple" problems can be surprisingly complex. The first half of this paper provides a conceptual (and almost chronological) development of DAGMan to illustrate the complexities that Condor deal with.

In the past several years, Condor has expanded it focus from running jobs on local clusters of computers (or pools, in Condor terminology) to running jobs in distributed grid environments. Along with the additional complexities in running jobs came greater challenges in transferring data to and from the job execution sites. We have developed Stork, which along with being a bird name, treats data placement with the same concern that Condor treats job execution.

With a combination of DAGMan, Condor, and Stork, users can create large, complex workflows that reliably "get the job done" in a grid environment. In the rest of this paper, we explore DAGMan and Stork (Condor has been covered in detail elsewhwere). 


\subsection{DAGMan Design Principles}

The goal of DAGMan is to automate the submission and management of complex workflows involving many jobs, with a focus on reliability and faulttolerance in the face of a variety of errors. Workflow management includes not only job submission and monitoring, but job preparation, cleanup, throttling, retry, and other actions necessary to ensure the good health of important workflows.

DAGMan attempts to overcome or work around as many errors as possible, and in the face of errors it cannot overcome, it endeavors to allow the user to resolve the problem manually and then resume the workflow from the point where it last left off. This can be thought of as a "checkpointing" of the workflow, just as some batch systems provide checkpointing of jobs.

Notably, the majority of DAGMan's features - and even some of its specific semantics - were not originally envisioned, but rather are the product of years of collaboration with active users. The experience gained from the needs and problems of "production" science applications has driven most DAGMan development over the past six years.

The fundamental design principles of DAGMan are as follows:

- DAGMan sits as a layer "above" the batch system in the software stack. DAGMan utilizes the batch system's standard API and logs to submit, query, and manipulate jobs, and does not directly interact with jobs independantly. ${ }^{1}$

- DAGMan reads the logs of the underlying batch system to follow the status of submitted jobs, rather than invoking interactive tools or service APIs. Reliance on simpler, file-based i/o allows DAGMan's own implementation to be simpler, more scalable and reliable across many platforms, and therefore more robust.

- DAGMan has no persistent state of its own - its runtime state is built entirely from its input files, and from the information gleaned by reading logs provided by the batch system about the history of the jobs it has submitted.

\footnotetext{
${ }^{1}$ Note that DAGMan assumes the batch system guarantees that it will not "lose" jobs after they have been successfully submitted. Currently, if the job is lost by the batch system after being successfully submitted by DAGMan, DAGMan will wait indefinitely for the status of the job in the queue to change. An explicit query for the status of submitted jobs (as opposed to waiting for the batch system to record job status changes) may be necessary to address this. Also, if a job languishes in the queue forever, DAGMan is currently not able to "timeout" and remove the job and mark it as failed. When removing jobs, detecting and responding to the failure of a remove operation (leaving a job "stuck" in the queue) is an interesting question.
} 


\subsection{DAGMan details}

\subsubsection{DAGMan Basics}

DAGMan allows users to express job dependencies as arbitrary directed acyclic graphs, or DAGs. In the simplest case, DAGMan can be used to ensure that two jobs execute sequentially - for example, that job B is not submitted until job A has completed successfully.

Like all graphs, a DAGMan DAG consists of nodes and arcs. Each node represents a single instance of a batch job to be executed, and each arc represents the execution order to be enforced between two nodes. Unlike more complex systems such as [21], arcs merely indicate the order in which the jobs must run.

If an arc points from node $N_{p}$ to $N_{c}$, we say that $N_{p}$ is the parent of $N_{c}$, and $N_{c}$ is the child of $N_{p}$. (See Figure 1.1.) A parent node must complete successfully before any of its child nodes can be started. Note that each node can have any whole number of parents or children (including zero). DAGMan does not require DAGs to be fully connected.

Why does DAGMan require a directed acyclic graph instead of an arbitrary graph? The graph is directed in order to express the order that jobs must run. Likewise, the graph is acyclic to ensure that DAGMan will not run indefinitely. In practice, we find that most workflows we encounter either do not require loops, or the loops can be unrolled into an acyclic graph.

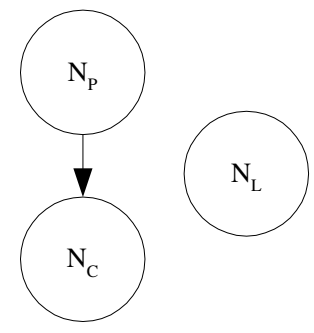

Fig. 1.1. The relationship between parents and children. $N_{p}$ is the parent of $N_{c}$. $N_{L}$ is lonely and has no parents or children.

DAGMan seeks to run as many jobs as possible in parallel, given the constraints of their parent/child relationships. For example, in for the DAG in Figure 1.2, DAGMan will initially submit both $N_{1}$ and $N_{5}$ to Condor, allowing them to execute in parallel if there are sufficient computers available. After $N_{1}$ completes successfully, DAGMan will submit both $N_{2}$ and $N_{3}$ to the batch system, allowing them to execute in parallel with each other, and with $N_{5}$ if it has not completed already. When both $N_{2}$ and $N_{3}$ have finished successfully, 
DAGMan will submit $N_{4}$. If $N_{5}$ and $N_{4}$ both complete successfully, the DAG will have completed, and DAGMan will exit successfully.

Earlier we defined a node's parent and child relationships. In describing DAGs, it can also be useful to define a node's sister as any node which shares the same set of parent nodes (including the empty set). Although sister relationships are not represented explicitly inside DAGMan, they are important because sister nodes always become "runnable" simultaneously, when their parents complete successfully. In Figure 1.2, $N_{1}$ and $N_{5}$ are sisters with no parents, and $N_{2}$ and $N_{3}$ are sisters that share $N_{1}$ as a parent.

In practice, however, DAGMan submits individual jobs to the batch scheduler one at a time, and makes no guarantees about the precise order that it will submit the jobs of nodes that are ready to run. In other words, $N_{1}$ and $N_{5}$ may be submitted to the batch system in any order.

It is also important to remember that, once submitted, the batch system is free to run jobs in its queue in any order it chooses. $N_{5}$ may run after $N_{4}$, despite being submitted to the queue earlier. Additionally, the jobs may not be run in parallel if there are insufficient compute resources for all parallel jobs.

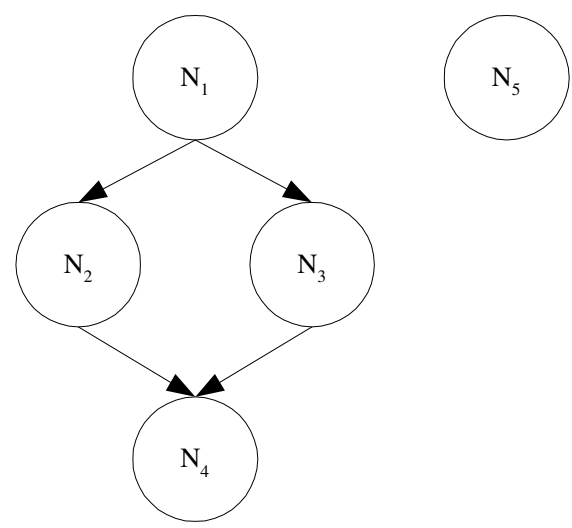

Fig. 1.2. This "diamond" dag illustrates parent and child links. $N_{1}$ must complete successfully, then both $N_{2}$ and $N_{3}$ can execute in parallel. Only when both of them have finished successfully can $N_{4}$ begin execution. $N_{5}$ is a disconnected node and can execute in parallel with all of the other nodes.

While running, DAGMan keeps a list in memory of all jobs in the DAG, their parent/child relationships, and their current status. Given this information, DAGMan submits jobs to the batch system when appropriate, and continues until either the DAG is complete, or no more forward progress can be made due to failed jobs. In the latter case, DAGMan creates a list of failed jobs along with the reasons for their failure, and produces a rescue $D A G$ file. 


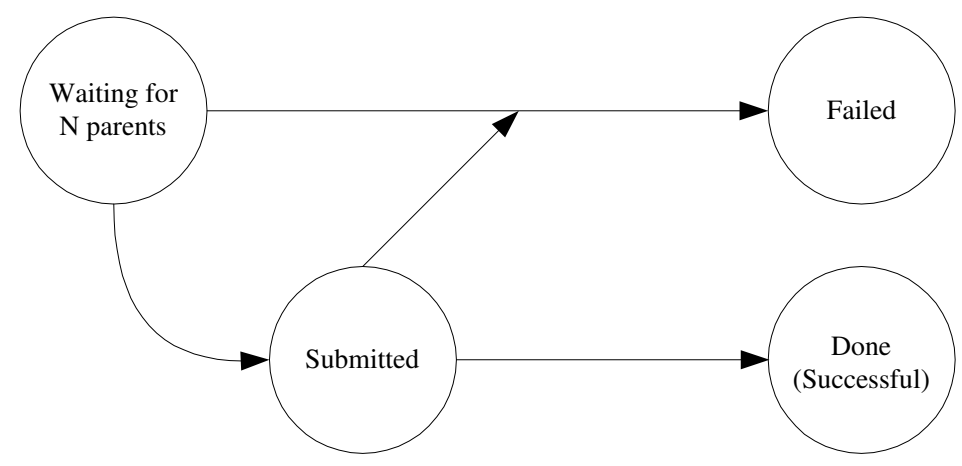

Fig. 1.3. The state transition diagram for each node of a DAG. See text for details.

A rescue DAG is a special DAG that represents the state of a previously partially-completed DAG such that the original DAG can be restarted where it left off, without repeating any successfully completed work. The rescue DAG is an exact copy of the original input DAG, except that all previously nodes that have successfully completed are marked as done.

When DAGMan is restarted with a rescue DAG, it reconstructs the state of the previous DAG. Internally, DAGMan keeps track of the current status of each node. Figure 1.3 shows the basic state diagram of a DAG node.

When DAGMan starts, it marks each node as "waiting", and initializes a waiting count $(\mathrm{N})$ for the node equal its number of parents. In the case of a rescue DAG, DAGMan sets the waiting count equal the number of parents which are not already marked as "done".

A node's waiting count represents the number of its parents that have yet to complete successfully, and which are therefore preventing it from being submitted. Only when a node's waiting count reaches zero can DAGMan submit the job associated with the node. If the job is submitted successfully, DAGMan marks the node as "submitted". If the job submission fails for any reason, the node is marked as "failed".

When DAGMan detects that a job has left the batch system queue, it marks the node as "done" if the job exited successfully, or oherwise marks it "failed". Success is determined by the exit code of the program: if it is zero, then the job exited successfully, otherwise it failed. (But see the description of post-scripts later in Section 1.3.2 for a modification of this.)

When a job is marked "done", the waiting count of all its children is decremented by one. Any nodes whose waiting count reaches zero is submitted to the batch scheduler as described earlier. 


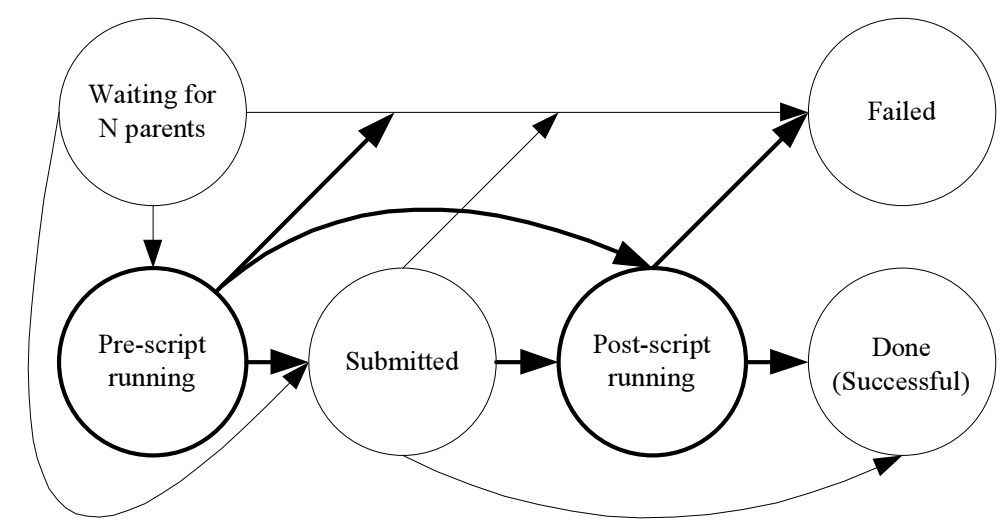

Fig. 1.4. A state diagram for executing a single DAG node. Unlike Figure 1.3, this diagram adds the ability to run pre-scripts and post-scripts. The differences from Figure 1.3 are noted in bold.

\subsubsection{DAGMan Complications}

So far, the description of a DAGMan DAG is not very interesting: we execute jobs and maintain the order in which they must execute, while allowing parallelism when it is possible. Unfortunately, this is insufficient in real environments, which have many complications and sources of errors.

\section{Complication: Setup, Cleanup, or Interpretation of a Node}

The first complication occurs when using executables that are not easily modified to run in a distributed computing environment and therefore need a setup or cleanup step to occur before or after the job. For example, before a job is run data may need to be staged from a tape archive or uncompressed. While this step could be placed in a separate DAG node, this may cause unnecessary overhead because the DAG node will be submitted and scheduled as a separate job by the batch system, instead of running immediately on the local computer.

DAGMan provides the ability to run a program before (a pre-script) or after (a post-script) a job is run. These programs should be lightweight because they are executed on the node from which the DAG was submitted and a large DAG may execute many of these scripts. (But see Section 1.3.2 for a way that DAGMan can deal with this.)

Running these scripts adds complexity to the state diagram in Figure 1.3. The changes needed to support scripts are shown in Figure 1.4. Once a job is allowed to run, it can optionally run a pre-script. After the job has run, it can optionally run a post-script. 


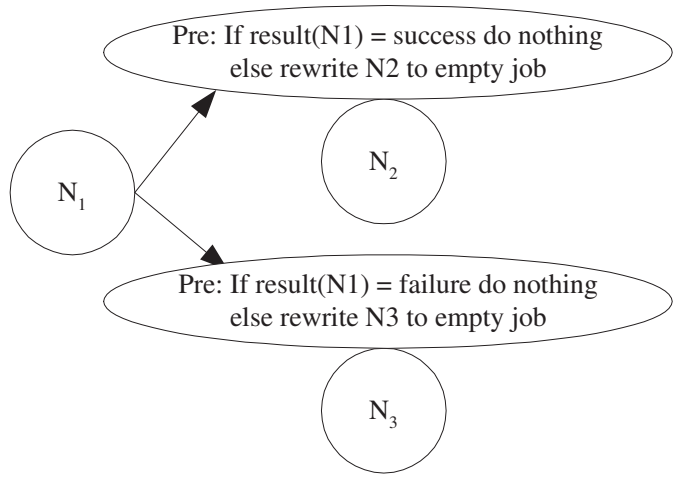

Fig. 1.5. An example conditional DAG.

Note that if the scripts fail, the node is considered to have failed, just as if the job itself had failed. There is one interesting case to note, which is not easily represented in Figure 1.4: if a node has a post-script, it will never directly go into the failed state, but will always run the post-script. In this way, the post-script can decide if a job has really failed or not. It can do some analysis beyond the DAGMan's ability decide if a node should be considered to have succeeded or failed based on the whether the exit code is zero or not. The significantly enhances the ability of DAGMan to work with exisiting code.

Some users have discovered an interesting way to use post-scripts. They create post-scripts that rewrite their child nodes' job description file to change how the job runs. This can be used for at least two purposes. First, it can create conditional DAGs by allowing a run-time decision that changes the DAG. For example, consider Figure 1.5. If $N_{1}$ succeeds, then the prescript for $N_{3}$ will rewrite $N_{3}$ to an empty job-perhaps running the /bin/true command. ${ }^{1}$ In this way, only $N_{2}$ will run after $N_{1}$ succeeds. Similarly, if $N_{1}$ fails, then only $N_{3}$ will run. While a more generic facility for conditional DAGs may be desirable, it would add complexity, and simple conditional DAGs can be created with .

A second use for pre-scripts is to do last-minute planning. For example, when submitting jobs to Condor-G (which allows jobs to be submitted to remote grid sites instead of the local batch system), users can specify exactly which grid site they wish their jobs to run at. A pre-script can decide what grid site should be used, rewrite the job description, and the job will run there.

\footnotetext{
${ }^{1}$ In recent versions of Condor, the job can be edited to contain " $n o o p_{-} j o b=$ true" which leaves the executable name alone, and immediately terminates the job successfully
} 


\section{Complication: Throttling}

All of the mechanisms described so far work very well. Unfortunately, the real world applies additional constraints. Imagine a DAG that can have one thousand jobs simultaneously running, and each of them has a pre-script and a post-script. When DAGMan can submit the jobs, it will start up one thousand nearly simultaneous pre-scripts, then submit one thousand jobs nearly simultaneously. Runing that many pre-scripts may cause an unacceptable load on the submission machine, and submitting that many jobs to the underlying batch submission system may also strain its capacity. For this reason, DAGMan can throttle the number of pre-scripts, jobs, or post-scripts that may run at any time. This results in another modification to our state diagram for running a single node, as shown in Figure 1.6.

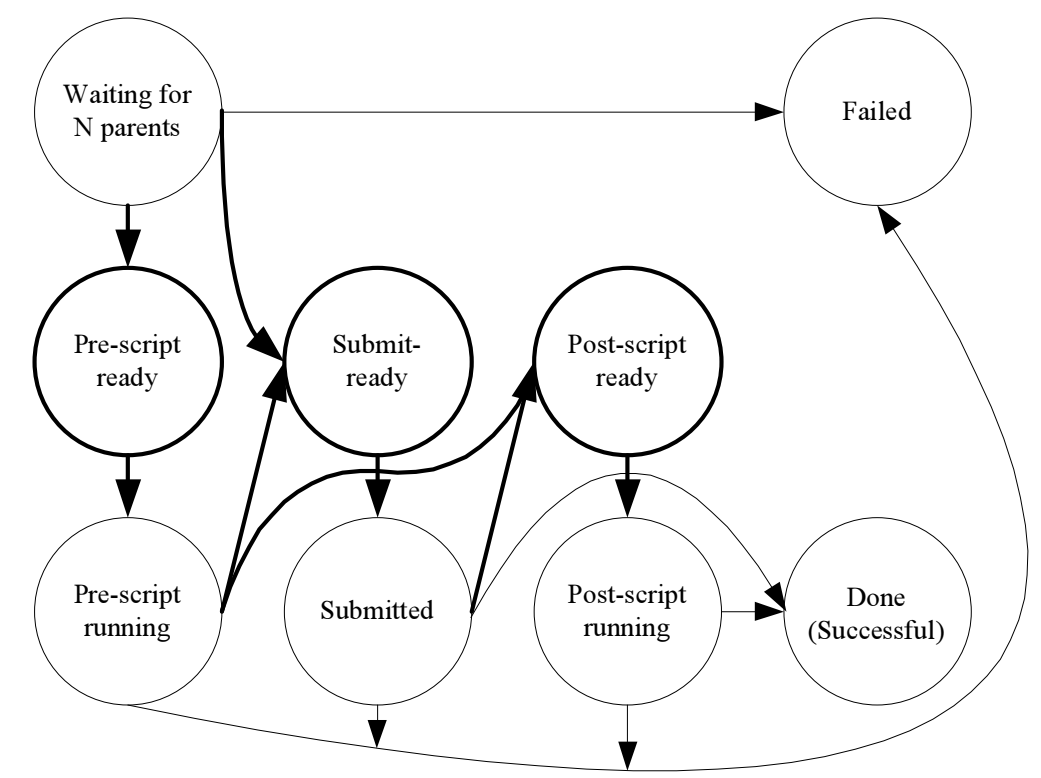

Fig. 1.6. A state diagram for executing a single DAG node. In addition to the state in Figure 1.4, this diagram adds DAGMan's ability to throttle pre-scripts, jobs, and post-scripts. The differences from Figure 1.4 are noted in bold.

DAGMan can also throttle the number of jobs that it submits to the batch system. This number might be significantly greater than the number of running job so this can prevent overloading the batch system. This is a good example of a surprising additional constraint: we did not realize that DAGs might be able to submit so many jobs that the number of idle jobs could overwhelm the batch system. 


\section{Complication: Unreliable applications or subsystems}

Some applications are not robust - it is not uncommon to find a program that sometimes fails to run on the first attempt, but completes successfully if given another chance. Sometimes it is due to a program error, sometimes due to interactions with the environment, such as a flaky networked file system. Ideally, problems such as this would always be fixed before trying to run the program. Unfortunately, this is not always possible, perhaps because the program is closed-source or because of time constraints.

To cope with unreliable programs or environments, DAGman provides the ability to retry a node if it fails. Users specify how many times the node should be retried before deciding that it has actually failed. When a node is retried, the node's pre-script is also run again. In some cases, a user wants to retry multiple times unless some catastrophic error occurs. DAGMan handle this with the "retry unless-exit" feature which will retry a job unless it exits with a particular value. One place this might be useful is planning: imagine a pre-script that decides where a job should be run. Retry might be set to 10, to allow the job to be run at ten different sites, but if there is some catastrophic error, then the pre-script can exit with a specific value that indicates "do not retry".

Adding the ability to retry the job results in one final change to our state diagram, as shown in Figure 1.7.

\subsubsection{Additional DAGMan Details}

We will briefly mention several other interesting DAGMan features.

\section{Running DAGMan robustly}

What happens if the machine on which DAGMan is running crashes? Although DAGMan would no longer continue to submit jobs, existing jobs continue running, but it would be nice if DAGMan could be restarted so that it could continue making forward progress. Ideally, DAGMan should handle as much as possible for the user, so we run DAGMan as a Condor job. This means that if the machine crashes, when it restarts Condor will restart the DAGMan process, which will recover the state of its execution from persistent log files, and will resume operation. This sort of robustness is essential in allowing users to run large sets of jobs in a "hands-off" fashion.

\section{Recursive DAGs}

A DAG node can do anything, including submitting another DAG. This allows for the creation of DAGs with conditional branches in them. The DAG node can make a choice, then submit an independent DAG based on the result of that choice. This can allow for very complex DAGs to be executed. 


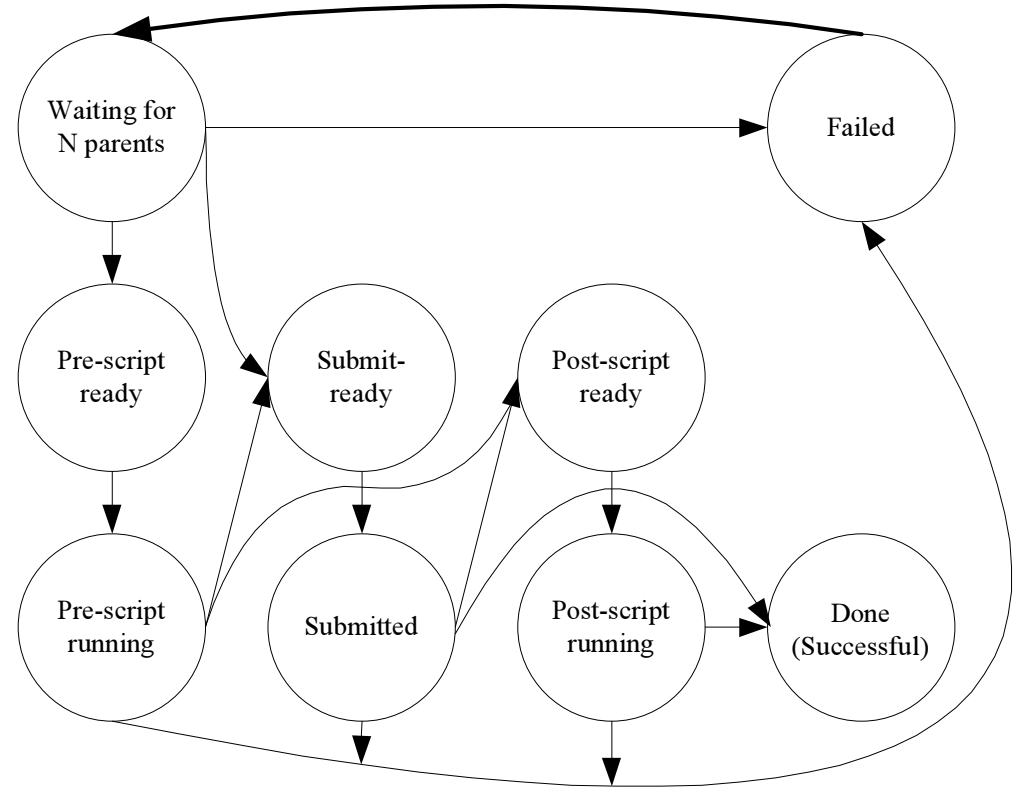

Fig. 1.7. The complete state diagram for executing a single DAG node. The single difference from Figure 1.6 is noted in bold.

Unfortunately, it also makes debugging a DAG harder. For an alternative to recursive DAGs, see Section 1.7.

\subsubsection{Describing a DAG}

It is the user's responsibility to provide DAGMan with a description of each job in the format of the underlying batch scheduler. For Condor, this means associating each node with a "submit file" describing the job to be executed. DAGMan ultimately uses this file to submit the job to the batch scheduler using the standard submission interface.

Users describe a DAG by listing each node and the relationships between nodes. A sample DAG description is shown in Figure 1.8.

\subsubsection{DAGMan Experience}

DAGMan has been used extensively with the Condor batch job scheduling system. In particular, we have used it for managing sets of jobs using BLAST for protein analysis, sets of jobs for simultation of events for high-energy physics, and many other uses. We have found that our implementation of the DAGMan easily scales to large DAGs of around 1000 nodes without throttling and DAGs of around 100,000 nodes with throttling. We believe it could scale 


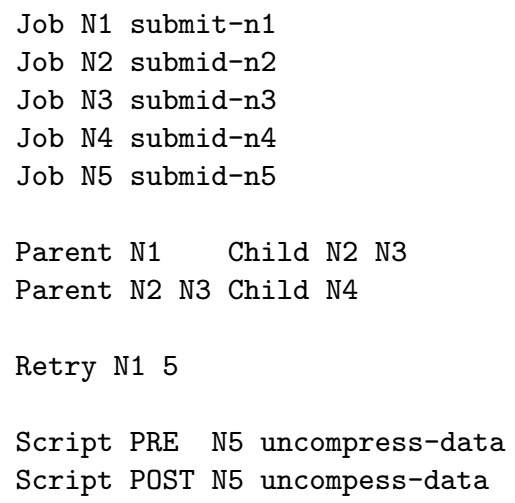

Fig. 1.8. How a user might describe the diamond dag from Figure 1.2. In this description, node $\mathrm{N} 1$ can be retried 5 times and all of the other nodes are not retried if they fail. Node N5 has both a pre-script and a post-script

much further than that if necessary. Because DAGMan can manage DAGs of this scale and because we find that the greatest bottleneck is in the underlying batch job submission systems capabilities, we have not expended effort to optimize it to work with larger DAGs.

DAGMan has been used in a wide variety of production environments. We will provide two examples here.

Within the Condor Project, we have created a BLAST [5] analysis service for the Biological Magentic Resonance Data Bank at the University of Wisconsin-Madison. [6] BLAST finds regions of local similarity between nucleotide or protein sequences. Local researchers do weekly queries against databases that are updated every week. Our service takes a list of sequences to query and creates a pair of DAGs to perform the queries, as illustrated in Figure 1.9. The first DAG performs the setup, the creation of a second DAG that does the queries (the number of nodes in this DAG varies, so it is dynamically created), and then assemble the results. These DAGs are used differently: the first DAG uses dependencies to order the jobs that are run, while the second DAG has completely independent nodes and DAGMan is used for reliable execution and throttling. On average, the second DAG has approximately 1000 nodes, but we have experimented with as many as 200,000 nodes. This service has run on a weekly basis for more than two years with little human supervision.

The Virtual Data System (VDS) [9] builds on top of DAGMan and CondorG. Users provide a description of what data is available and how the data can be transformed, then request the data they need. The VDS creates a DAG that fetches and transforms data as needed, while tracking the provenance of the data. As part of the DAG creation and execution, the VDS uses planning to decide which grid sites should perform the transformations. The VDS has 

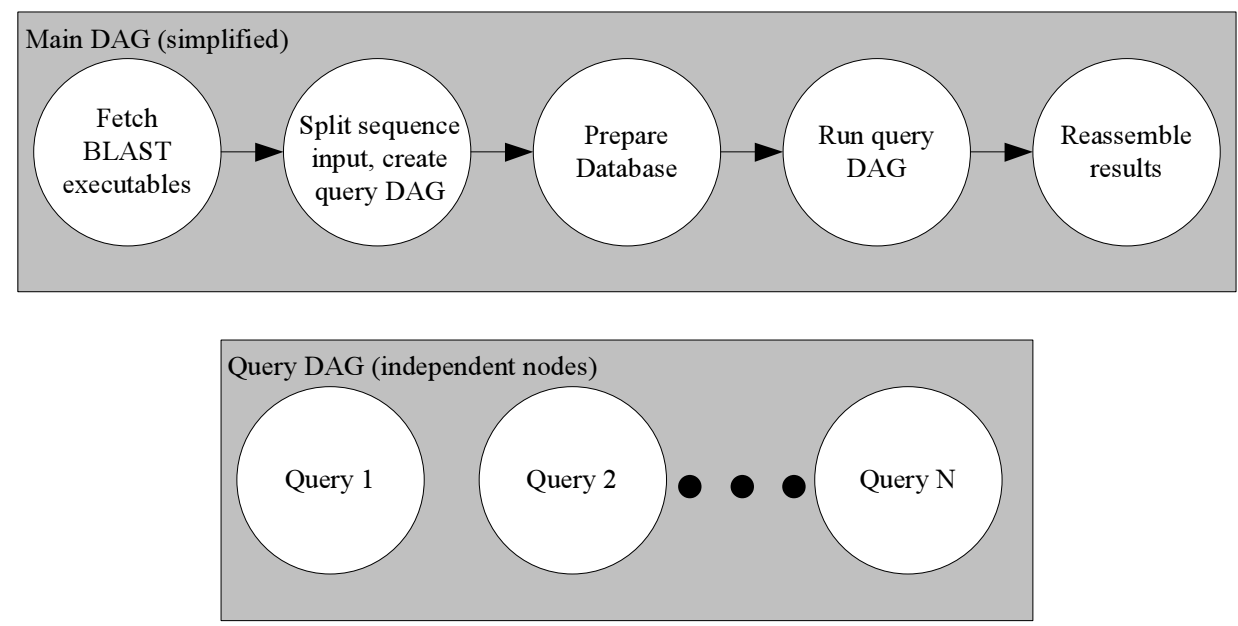

Fig. 1.9. The pair of DAGs used to run BLAST jobs. The query DAG is created by the main DAG. See text for details.

been used for a wide variety of applications including high-energy physics event simulation, galaxy cluster finding, and genome analysis.

\subsection{Implementation Status}

DAGMan has been freely distributed as part of the Condor software since 1999. It has been used for numerous large projects, and is stable. It is available for a wide variety of Unix platforms, and Microsoft Windows.

\subsection{Interaction with Condor}

Condor is a high-throughput batch job scheduler. Because it has been covered in detail elsewhere([17], [20]), we only briefly review it here.

Condor was originally designed to utilize CPU cycles on computers that would otherwise be idle, such as desktop computers that are unused but turned on overnight. However, Condor has expanded its reach and now works well with dedicated computers and grid systems. Condor's ability to interact with grid system, called Condor-G [10], allows Condor to submit jobs to Globus [11] (versions 2, 3, and 4), NorduGrid, Oracle, LSF, PBS, and even remote Condor installations (referred to as Condor-C).

Condor and Condor-G emphasize reliability. If Condor crashes, it will continue running the jobs when it restarts. Condor can provide job checkpointing and migration to facilitate recover when execution computers fail. Condor-G 
provides elaborate recovery schemes to deal with network outages and remote grid site failures.

DAGMan is built to use Condor for job execution, and it can submit jobs to both the local batch system and remote grid systems with equal ease. We have created many workflows using DAGMan that execute in a grid environment.

\subsection{Integration with Stork}

\subsubsection{An Introduction to Stork}

Just as computation and network resources need to be carefully scheduled and managed, the scheduling of data placement activities all across the distributed computing systems is crucial because the access to data is generally the main bottleneck for data intensive applications. This is especially the case when most of the data is stored on tape storage systems, which slows down access to data even further due to the mechanical nature of these systems.

The current approach to solve this problem of data placement is either doing it manually, or employing simple scripts, which do not have any automation or fault tolerance capabilities. They cannot adapt to a dynamically changing distributed computing environment. They do not have the privileges of a job, they do not get scheduled, and generally require baby-sitting throughout the process.

Data placement activities must be first class citizens in the distributed computing environments just like the computational jobs. They need to be queued, scheduled, monitored, and even check-pointed. More importantly, it must be made sure that they complete successfully and without any need for human intervention.

Moreover, data placement jobs should be treated differently from computational jobs, since they have different semantics and different characteristics. For example, if the transfer of a large file fails, we may not simply want to restart the job and re-transfer the whole file. Rather, we may prefer transferring only the remaining part of the file. Similarly, if a transfer using one protocol fails, we may want to try other protocols supported by the source and destination hosts to perform the transfer. We may want to dynamically tune up network parameters or decide concurrency level for specific source, destination and protocol triples. A traditional computational job scheduler does not handle these cases. For this purpose, data placement jobs and computational jobs should be differentiated from each other and each should be submitted to specialized schedulers that understand their semantics.

We have designed and implemented the first batch scheduler specialized for data placement: Stork [16]. This scheduler implements techniques specific to queuing, scheduling, and optimization of data placement jobs, and provides a level of abstraction between the user applications and the underlying data transfer and storage resources. 
A production level Stork is bundled with Condor releases. Additionally, research into new features is continuing in parallel.

\subsubsection{Data Placement Job Types}

Under Stork, data placement jobs are catagorized into the three following types:

transfer This job type is for transferring a complete or partial file from one physical location to another one. This can include a get or put operation or a third party transfer. Stork supports a variety of data transfer protocols, including: local file system, GridFTP, FTP, HTTP, NeST, SRB, SRM and UniTree. Further, sites can create new transfer modules using the Stork modular API.

allocate This job type is used for allocating storage space at the destination site, allocating network bandwidth, or establishing a light-path on the route from source to destination. Basically, it deals with all necessary resource allocations pre-required for the placement of the data.

release This job type is used for releasing the corresponding resource which is allocated before.

\subsubsection{Flexible Job Representation}

Stork uses the ClassAd [19] job description language to represent the data placement jobs. The ClassAd language provides a very flexible and extensible data model that can be used to represent arbitrary services and constraints.

Below are three sample data placement $(\mathrm{DaP})$ requests:

[

dap_type = "allocate";

dest_host = "houdini. example.com";

size $\quad=$ "200MB";

duration $\quad=$ "60 minutes";

]

allocation_id = 1 ;

[

dap_type $=$ "transfer";

src_url = "file:////data/example.dat";

]

dest_url = "gsiftp://houdini.example.com/data/example.dat";

[

dap_type = "release";

dest_host $\quad=$ "houdini.example.com";

]

allocation_id = 1 ;

The first request is to allocate $200 \mathrm{MB}$ of disk space for 1 hour on a NeST server. The second request is to transfer a file from an SRB server to the allocated space on the NeST server. The third request is to de-allocate the previously allocated space. 


\subsubsection{Fault Tolerance}

Data placement applications must operate in an environment of faults. Data servers may be unavailable for many reasons. Remote and local networks may encounter outages or congestion. host on the local area network, including the host running Stork. Stork is equipped to deal with a variety of data placement faults, which can be configured at both the system, and job level.

For transient environment faults, data placement jobs that fail can be retried after a small delay. The number of retries allowed is a system configuration.

For longer term faults associated with a particular data server Stork can also retry a failed transfer using a list of alternate data protocols. If in the previous example, the host ghidorac.sdsc.edu is also running a GridFTP server, accessed via the gsiftp protocol, the corresponding transfer job could be augmented to retry a transfer failure from the primary SRB to NeST protocols, with a transfer attempt from the gsiftp to NeST protocols:

[

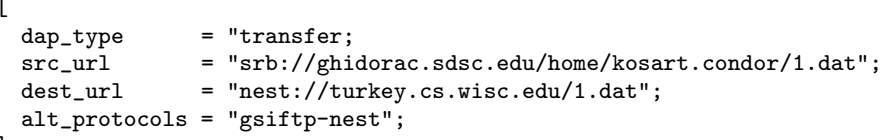

\subsubsection{Interaction with DAGMan}

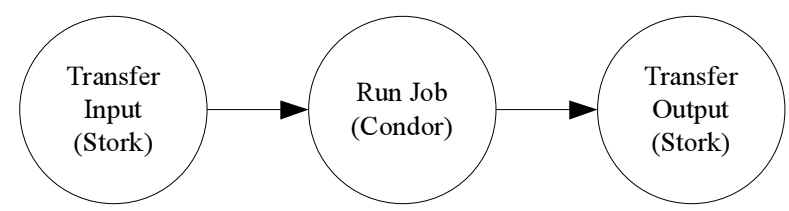

Fig. 1.10. A simple DAG that includes Stork

DAGMan has been extended to work well with Stork. In addition to specifying computational jobs, data placement jobs can be specified, and DAGMan will submit them to Stork for execution. This allows for straightforward execution of workflows that include data transfer.

A simple example of how Stork can be used with DAGMan appears in Figure 1.10. This DAG transfers data to a grid site, executes the job at that site, then transfers the output data back to the submission site. This DAG could easily be enhanced to allow space allocation before the data transfers, or could have multiple data transfers. 


\subsubsection{Interaction with Heterogeneous Resources}

Stork acts like an I/O control system (IOCS) between the user applications and the underlying protocols and data storage servers. It provides complete modularity and extendibility. The users can add support for their favorite storage system, data transport protocol, or middleware very easily. This is a very crucial feature in a system designed to work in a heterogeneous distributed environment. The users or applications may not expect all storage systems to support the same interfaces to talk to each other. And we cannot expect all applications to talk to all the different storage systems, protocols, and middleware. There needs to be a negotiating system between them which

can interact with those systems easily and even translate different protocols to each other. Stork has been developed to be capable of this. The modularity of Stork allows users to insert a plug-in to support any storage system, protocol, or middleware easily.

Stork supports several data transfer protocols, including:

- FTP [18]

- GridFTP [1]. Stork supports third-party data transfer with GridFTP, eliminating the need for local storage.

- $\operatorname{HTTP}[8]$

- DiskRouter [14]

Stork supports several data storage systems, including:

- $\quad$ SRB [3]

- UniTree [7]

- NeST [4]

Stork maintains a library of pluggable "data placement" modules. These modules get executed by data placement job requests coming into Stork. They can perform inter-protocol translations either using a memory buffer or thirdparty transfers whenever available. Inter-protocol translations are not supported between all systems or protocols yet.

In order to transfer data between systems for which direct inter-protocol translation is not supported, two consecutive Stork jobs can be used instead. The first Stork job performs transfer from the source storage system to the local disk cache of Stork, and the second Stork job performs the transfer from the local disk cache of Stork to the destination storage system.

\subsubsection{Modular API}

While the Stork server is a single process, the data transfers, allocations, etc, are performed by separate modules. The application program interface to the modules is simple enough for sites to write their own modules as needed. For example, each data transfer module is executed with the following argument list: 
src_url dest_url arguments ...

Thus, to write a new module that transfers data from the foo protocol to the bar protocol, a new module is created with the the name: stork.transfer.foo-bar. Modules are executable programs and may be written as shell scripts. Further, module binding is performed at runtime, enabling sites to create new modules without restarting the Stork server.

\subsubsection{Performance Enhancements}

Stork has seen several recent performance enhancements. The first is that Multiple data placements may now be specified in a single submission file instead of multiple files. This optimization is significant when transfering many files to Stork because it eliminates extra invocations of the stork $\searrow_{-}$submit command which can be surprisingly time consuming when transferring tens of thousands of files.

The second enhancement was integration of the GridFtp client, globus-url-copy into Stork server. When doing many simultaneous GridFTP file transfers, this saves considerable time and reduces the total number of processes in the system.

Finally, Stork is now able to execute an arbitrary program when the active job queue size falls below a configurable level. This is envisioned as a simple, but high performance alternative to managing very large data placement work flows with DAGMan because it will allow Stork users to limit the rate at which they submit jobs so that Stork is not overwhelmed, while ensuring that Stork has sufficient work to do at any given time.

\subsubsection{Implementation Status}

Stork is available, with all features described so far, as part of the Condor distribution. It is available on Linux, and will be available for other platforms in the future. Users outside of the Condor Project are just beginning to use Stork in production, and we hope to have more in the near future.

\subsubsection{Active Research}

Research on Stork is active, and much of it can be found in [15]. Research includes:

- Extending data placement types to include interaction with a metadata catalog.

- Experimentation with scheduling techniques other than first-in, first-out. This includes not only traditional scheduling techniques such as shortestjob first or multilevel queue priority scheduling, but also includes scheduling based on management of storage space to ensure that storage space is not exceeded by the data transfers. In addition, scheduling of connection 
management is important when there are many simultaneous connections to a server.

- Runtime adaption can be performed to tune the network parameters for a transfer to minimize transfer time. This is discussed in further detail in $[13]$.

- Research has been done to enable Stork to detect problems such as servers that are unreliable in a variety of ways, then base scheduling decisions on this knowledge. More details are in [12].

\subsection{Future Directions}

There are several promising areas for future work with DAGMan and Stork.

For DAGMan, we would like to explore methods (probably utilizing ClassAds [19]) to allow different conditions for deciding when a node should execute. Today, a node executes when all of its parents have finished with an exit code of 0 , but allowing more complex conditions would allow conditional execution (equivalent to if-then-else) and partial execution (a DAG that finishes when a certain percentage of nodes have completed.)

We also would like to support dymamic DAGs, which are DAGs that can change on-the-fly, based on user input. This has been frequently requested by users, and is particularly useful when DAGMan is used by a higher-level scheduling system that may change plans in reaction to current conditions.

For Stork, we are exploring ways to make it more scalable and more reliable. We are also investigating methods to make using matchmaking, similar to that in Condor, to select which data transfers should be run and which sites they should transfer data too.

\subsection{Conclusions}

DAGMan is a reliable workflow management system. Although the workflows it supports are relatively simple, there are many complexities that were discovered as we used DAGMan through the years, such as the need for flexible methods for retrying and throttling. As a result of our experience, DAGMan has found favor with many users in production environments, and software has been created that relies on DAGMan for execution. Used with Condor, Condor-G, and Stork, DAGMan is a powerful tool for workflow execution in grid environments. 


\section{References}

1. W. Allcock, J. Bester, J. Bresnahan, A. Chervenak, I. Foster, C. Kesselman, S. Meder, V. Nefedova, D. Quesnel, and S. Tuecke. Data management and transfer in high-performance computational grid environments. Parallel Computing, 28(5):749-771, May 2002.

2. A. Baker. The Secret Ways of Al Baker. The Miracle Factory, 2003.

3. C. Baru, R. Moore, A. Rajasekar, and M. Wan. The SDSC storage resource broker. In Proceedings of CASCON, 1998.

4. J. Bent, V. Venkataramani, N. LeRoy, A. Roy, J. Stanley, A. Arpaci-Dusseau, R. Arpaci-Dusseau, and M. Livny. NeST - a grid enabled storage appliance. In J. Weglarz, J. Nabrzyski, J. Schopf, and M. Stroinkski, editors, Grid Resource Management. Kluwer Academic Publishers, 2003.

5. Basic local alignment search tool (BLAST). http://www.ncbi.nlm.nih.gov/ blast/, 2006.

6. Biological magnetic resonance data bank. http://www.bmrb.wisc.edu/, 2006.

7. M. Butler, R. Pennington, and J. A. Terstriep. Mass storage at NCSA: SGI DMF and HP UniTree. In Proceedings of 40th Cray User Group Conference, 1998.

8. R. Fielding, J. Gettys, J. Mogul, H. Frystyk, L. Masinter, P. Leach, and T. Berners-Lee. Hypertext transfer protocol - HTTP/1.1. Internet RFC 2616, Jun 1999.

9. I. Foster, J. Voeckler, M. Wilde, and Y. Zhao. Chimera: A virtual data system for representing, querying, and automating data derivation. In 14th International Conference on Scientific and Statistical Database Management (SSDBM'02), 2002.

10. J. Frey, T. Tannenbaum, I. Foster, M. Livny, and S. Tuecke. Condor-G: A computation management agent for multi-institutional grids. Cluster Computing, 5:237-246, 2002.

11. The Globus Alliance. See website at http://www.globus.org.

12. G. Kola, T. Kosar, and M. Livny. A client-centric grid knowledgebase. In Proceedings of 2004 IEEE International Conference on Cluster Computing, pages 431-438, San Diego, CA, September 2004. IEEE.

13. G. Kola, T. Kosar, and M. Livny. Run-time adaptation of grid data-placement jobs. Parallel and Distributed Computing Practices, 2004. 
14. G. Kola and M. Livny. Diskrouter: A flexible infrastructure for high performance large scale data transfers. Technical Report CS-TR-2003-1484, University of Wisconsin-Madison Computer Sciences Department, 2003.

15. T. Kosar. Data Placement in Widely Distributed Systems. PhD thesis, University of Wisconsin-Madison, 2005.

16. T. Kosar and M. Livny. Stork: Making data placement a first class citizen in the grid. In Parallel and Distributed Computing Practices, 2004.

17. M. Litzkow, M. Livny, and M. Mutka. Condor - a hunter of idle workstations. In Proceedings of the 8th International Conference on Distributed Computing Systems, pages 104-111, June 1988.

18. J. Postel and J. Reynolds. File transfer protocol (FTP). Internet RFC 959, Oct 1985.

19. R. Raman, M. Livny, and M. Solomon. Matchmaking: Distributed resource management for high throughput computing. In Proceedings of the Seventh IEEE International Symposium on High Performance Distributed Computing (HPDC7), Chicago, IL, July 1998.

20. D. Thain, T. Tannenbaum, and M. Livny. Distributed computing in practice: the condor experience. Concurrency - Practice and Experience, 17(2-4):323-356, 2005.

21. W. Woods. What's in a link: Foundations for semantic networks. In D. Bobrow and A. Collins, editors, Representation and Understanding: Studies in Cognitive Science. New York: Academic Press, 1975. 